\title{
The relationship between the severity of clinical symptoms of chronic urticaria and serum D-dimer levels
}

\author{
Anna Sadowska-Przytocka ${ }^{1}$, Magdalena Czarnecka-Operacz ${ }^{1}$, Katarzyna Łącka², Dorota Jenerowicz ${ }^{1}$, Zygmunt Adamski $^{1}$ \\ ${ }^{1}$ Department of Dermatology, Poznan University of Medical Sciences, Poznan, Poland \\ 2Department of Endocrinology, Metabolism and Internal Medicine, Poznan University of Medical Sciences, Poznan, Poland \\ Adv Dermatol Allergol 2021; XXXVIII (3): 486-489 \\ DOI: https://doi.org/10.5114/ada.2020.94594
}

\begin{abstract}
Introduction: Urticaria and angioedema belong to a heterogeneous group of diseases and it is often very difficult to determine the specific cause of clinical symptoms. Coagulation factors play an important role, and patients with chronic urticaria have elevated plasma concentrations of coagulation factor VII, prothrombin fragment $1+2$ and D-dimers.

Aim: To assess the relationship between the severity of clinical symptoms of chronic urticaria and serum D-dimer levels.

Material and methods: Sixty patients with a diagnosis of chronic spontaneous urticaria, diagnosed and treated in the Dermatology Department of the Poznan University of Medical Sciences were qualified for the tests. The control group consisted of 35 healthy volunteers. All patients with chronic spontaneous urticaria were previously subjected to individual stages of the diagnostic process, in accordance with the guidelines of EAACI and the Polish team of specialists in the field of dermatology and venereology, and allergology. The dermatological condition was assessed using the UAS scale. The serum D-dimer concentration was determined by immunoturbidimetry.

Results: In the basic group, statistically significant, positive and high correlations between the serum D-dimer concentration and the severity of urticaria symptoms were shown, as well as between the severity of pruritus and the value of the disease activity index were shown.

Conclusions: Elevated levels of D-imers are significantly correlated with the severity of urticaria.
\end{abstract}

Key words: urticaria, D-dimers, treatment.

\section{Introduction}

According to the definition of the European Academy of Allergy and Clinical Immunology (EAACI), the Global Allergy and Asthma European Network (GA²LEN), the European Dermatology Forum (EDF) and the World Allergy Organization (WAO), urticaria is a heterogeneous group of diseases whose characteristic common clinical manifestation is specific skin lesions, which may be accompanied by symptoms of angioedema [1]. It is believed that as much as $12-25 \%$ of the world's population has developed urticaria at least once during their lifetime [2]. Of course, in the case of discrete clinical symptoms of urticaria, this reaction is not recorded by patients, hence the actual percentage of urticaria may be higher.

Activation of mast cells and the release of mediators from these cells is a common feature of most types of

urticaria. Mast cells are located in the immediate vicinity of blood vessels. Mast cells release both preformed mediators (e.g. histamine, heparin) and mediators produced immediately after activation - de novo (e.g. prostaglandins, leukotrienes) and cytokines (e.g. tumour necrosis factor $\alpha$, interleukin-8) [3]. Moreover, activation of the complement and release of the C5a fragment stimulates mast cells and acts chemotactically on neutrophils, eosinophils and monocytes [4]. As is known, stimuli and mediators act on the mast cell through receptors located within the cell membrane. Histamine, which is one of the basic mediators of urticaria reactions, can be released both in the immune mechanism and with the participation of mechanisms unrelated to the immune system. That is why urticaria and angioedema belong to a het-

Address for correspondence: Anna Sadowska-Przytocka, Department of Dermatology, Poznan University of Medical Sciences, 49 Przybyszewskiego St, 60-355 Poznan, Poland, phone: +48 661964 960, e-mail: a.sadowska80@gmail.com Received: 13.01 .2020 , accepted: 3.02.2020. 
erogeneous group of diseases and it is often very difficult to determine the specific cause of clinical symptoms.

Diagnosis of urticaria often poses a great clinical problem for practitioners. The test used in the diagnosis of chronic spontaneous urticaria (CSU) is an autologous serum skin test (ASST) to show autoreactivity. However, some authors question the specificity of ASST, pointing to the possibility of obtaining false positive results due to the release of large amounts of bradykinin during the clotting process and the breakdown of C 5 factor. The frequency of positive ASST results in patients with CSU ranges from $4.1 \%$ to $76.5 \%$ depending on the validation criteria used. According to Asero et al., the percentage of autoreactivity increases when using an autologous plasma skin test (APST). As much as $86 \%$ of patients obtained a positive APST in their study. Interestingly, a positive result was found in patients whose ASST was negative. In contrast, $98 \%$ of patients obtained a positive result for both ASST and APST. Different results were obtained in another multicentre study in which $37.5 \%$ had positive ASST results, while $43 \%$ had positive APST results. It seems that coagulation factors play an important role in the development of a positive skin reaction in APST, and in patients with chronic urticaria an elevated plasma concentration of coagulation factor VII, prothrombin fragment $1+2$ and D-dimers is known. Activation of the extrinsic coagulation cascade may be caused by the release of tissue factor, whereas according to Asero studies, the levels of plasma coagulation factors correlated with the severity of the disease process at the time of collecting blood for testing [5-7].

\section{Aim}

The aim of the study was to assess the relationship between the severity of clinical symptoms of chronic urticaria and serum D-dimer levels.

\section{Material and methods}

Sixty patients diagnosed and treated for CSU in the Dermatology Department of the Poznan University of Medical Sciences were qualified for tests. The patients' age ranged between 13 and 84 years, and the average age was 42.4 (median: 45 ). The group of patients examined comprised $76.7 \%$ of females (46 patients) and $23.3 \%$ of males (14 patients). This group was designated as "primary".

Control group II consisted of 35 healthy volunteers aged from 23 to 67 . The average age in this group was 38.7 (median: 33). Women constituted 62.9\% (22 persons) of the study group, while men constituted $37.1 \%$ (13 persons). In the interview for volunteers, there was no load of CSU clinical symptoms.

All patients with autoimmune CSU were previously subjected to individual stages of the diagnostic process, in accordance with the guidelines of EAACl and the Polish team of specialists in the field of dermatology and venereology and allergology. All patients included in the study were interviewed in depth, with particular regard to the following elements: the time of onset of the first symptoms of the disease, family burden of urticaria and/ or angioedema, atopic diseases, the presence of internal diseases, autoimmune diseases, medications used, food consumed, occupation and non-professional interests (hobby). Following this, a classical physical examination and assessment of the dermatological condition was carried out, together with an assessment of the severity of the disease process, which was done using the UAS (urticaria activity score) (Table 1) [1].

The serum D-dimer concentration was determined by immunoturbidimetry using the Tina-quant test from Roche Diagnostics (Switzerland). This method involves the reaction of anti-D monoclonal antibodies, located on a latex medium, with D-dimers contained in the tested plasma. The addition of a suspension of latex particles coated with serum antibodies with D-dimers causes agglutination manifesting in turbidity. Next, the absorbance of $540 \mathrm{~nm}$ is measured in the solution. Agglutinates formed as a result of the immune reaction have a larger diameter than the wavelength, hence the increase in wave absorption proportional to the increase in D-dimer concentration in the serum tested. Latex particles with antibodies that are not linked to the antigen have a smaller diameter to the wavelength passing through the solution and do not affect the result of the assay. The concentration of D-dimers $0-0.5 \mathrm{mg} / \mathrm{l}$ was taken as the reference value.

\section{Results}

The average UAS value in the population studied (basic group) was 2.72. The average number of urticaria wheals is 1.25 , while the average value of the severity index of itching was 1.47. Patients were classified on the basis of the number of points obtained in the clinical evaluation carried out on the UAS scale to 4 groups. In 47 (78.3\%) people, the clinical condition in relation to urticaria was symptomatic, i.e. wheals were present. Thirteen (21.7\%) patients were asymptomatic for both urticaria and angioedema.

The serum concentrations of D-dimers in the primary group were in the range of $0.1-4.4 \mathrm{mg} / \mathrm{l}$. In contrast, the mean concentration was $0.97 \mathrm{mg} / \mathrm{l}$ (median: 0.47). In the basic group, statistically significant, positive, high correlations between the serum D-dimer concentration and the severity of urticaria symptoms, pruritus severity and disease activity index value were demonstrated (Figures 1-3).

In the control group (healthy volunteers), the concentration of D-dimers was within the normal range. More- 
Table 1. Urticaria activity score

\begin{tabular}{lcc}
\hline Itch severity score & $\begin{array}{c}\text { Number of } \\
\text { hives per 24 h }\end{array}$ & $\begin{array}{c}\text { Hives severity } \\
\text { score }\end{array}$ \\
\hline 0 - None & None & 0 \\
\hline $\begin{array}{l}\text { 1- Mild (present but not } \\
\text { annoying or troublesome) }\end{array}$ & $<20$ & 1 \\
\hline $\begin{array}{l}\text { 2-Moderate (troublesome but } \\
\text { does not interfere with normal } \\
\text { daily activity or sleep) }\end{array}$ & $20-50$ & 2 \\
\hline $\begin{array}{l}3-\text { intense (interferes with } \\
\text { normal daily activity or sleep) }\end{array}$ & $>50$ & 3 \\
\hline
\end{tabular}

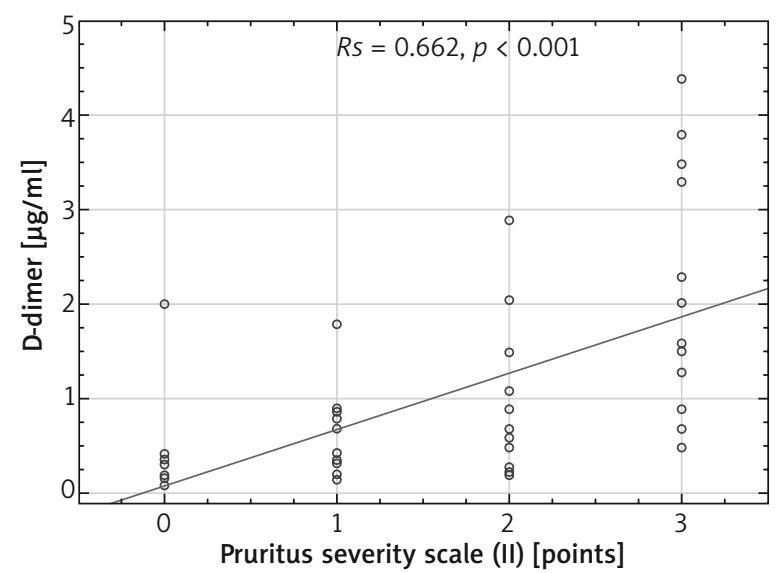

Figure 2. Relationship between D-dimer concentration and pruritus severity (pruritus severity scale $-\mathrm{II}$ ) in patients of the primary group

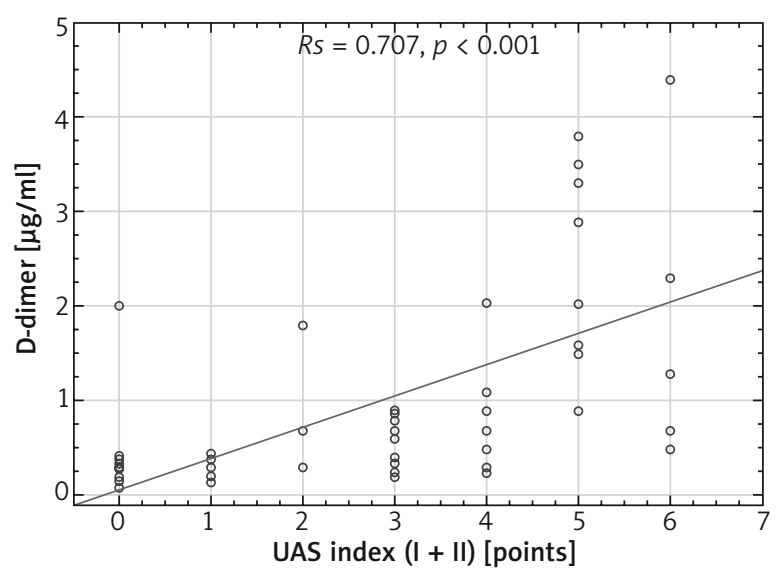

Figure 3. Relationship between D-dimer concentration and UAS index in patients of the primary group

over, these people did not show symptoms of urticaria and did not report pruritus in the subject examination.

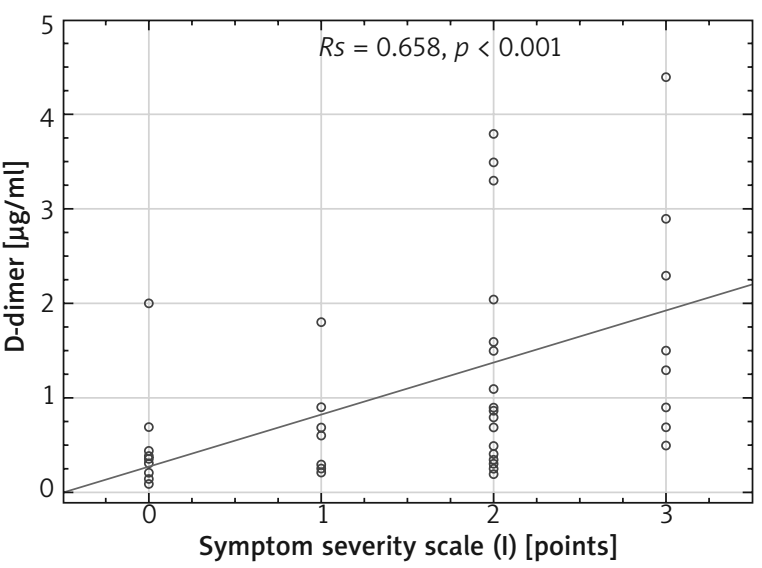

Figure 1. Relationship between D-dimer concentration and symptom severity (symptom severity scale $-\mathrm{I}$ ) in patients of the primary group

\section{Discussion}

The complex pathogenesis of urticaria and angioedema is also associated with the activation of the coagulation system. It was found that mast cells may exhibit pro-lipolytic activity. They are also responsible for the conversion of plasminogen to plasmin. In their research, Schwartz et al. showed that it is tryptase contained in mast cells that has fibrinolytic properties [8]. Other studies, in turn, have proved the effect of mast cells on prolonging bleeding time and inhibiting the formation of thrombin. In the therapeutic aspect, the results of a study demonstrating the clinical effectiveness of warfarin in the treatment of patients with CSU are interesting [9]. Importantly, heparin inhibited the formation of a positive skin reaction in the ASST procedure [10]. It shows that patients diagnosed with various autoimmune diseases have elevated levels of D-dimers, which results from the simultaneous activation of coagulation and fibrinolysis. Increased levels of D-dimers are associated with increased platinum processing of fibrin. What is also significant is that D-dimers are also responsible for the release of histamine from mast cells [11, 12]. It seems that markers of haemostasis can be useful in monitoring the activity of the disease process in the course of CSU and the effects of its treatment. Asero showed that D-dimer levels are elevated in patients not responding to the use of LP in maximum quadruple doses [13]. Such a correlation was found in 18 out of 23 patients, in whom no improvement was seen after the inclusion of $10 \mathrm{mg} /$ day cetirizine. The dose was increased 2- or 3-fold in those who did not improve. Then, patients were evaluated according to the UAS scale.

In our study, D-dimers were in the range of 0.1-4.4 $\mathrm{mg} / \mathrm{l}$. Their average level was $0.97 \mathrm{mg} / \mathrm{l}$. Statistically significant correlations were found between D-dimer levels and the severity of clinical symptoms (value according to 
the severity of urticaria symptoms), as well as between the severity of pruritus and disease activity (disease activity index value).

\section{Conclusions}

Based on the results of research conducted in this area, it can be concluded that elevated levels of D-dimers as well as other indicators of the acute phase of inflammation are significantly correlated with the severity of urticaria, but their actual importance in the pathogenesis of urticaria remains controversial and requires further multicentre studies on a larger patient population.

\section{Conflict of interest}

The authors declare no conflict of interest.

\section{References}

1. Zuberbier T, Aberer W, Asero R, et al. The EAACI/GA(2) LEN/ EDF/WAO Guideline for the definition, classification, diagnosis, and management of urticaria: the 2013 revision and update. Allergy 2014; 69: 868-87.

2. Rudzki E, Rebandel P. Częstość występowania poszczególnych odmian pokrzywki przewlekłej. Alergia Astma Immunol 2006; 11 supl. I: 246.

3. Poonawalla T, Kelly B. Urticaria: a review. Am J Clin Dermatol 2009; 10: 9-21.

4. Taneda K, Tominaga M, Tengara S, et al. Neurotropin inhibits both capsaicin-induced substance $P$ release and growth factor-induced neurite outgrowth In cultured rat dorsal root ganglion neurons. Clin Exp Dermatol 2010; 35: 73-7.

5. Asero R, Tedeschi A, Riboldi P, et al. Plasma of patients with chronic urticaria shows signs of thrombin generation, and its intradermal injection causes wheal- and-flare reactions much more frequently than autologous serum. J Allergy Clin Immunol 2006; 117: 1113-7.

6. Asero R, Cugno M, Tedeschi A. Chronic idiopathic urticaria: what is the meaning of skin reactivity to autologous serum? J Eur Acad Derm Venereol 2008; 22: 135-36.

7. Kasperska-Zajac A, Brzoza Z. Increased D-dimer concentration in plasma of patients with severe acute urticaria. Br J Dermatol 2009; 161: 1409-10.

8. Schwartz LB, Bardford TR, Littman BH, Wintroub BU. The fibrinogenolytic activity of purified tryptase from human lung mast cells. J Immunol 1985; 135: 2762-7.

9. Mahesh PA, Pudupakkam VK, Holla AD, Dande T. Effect of warfarin on chronic idiopathic urticaria. Indian J Dermatol Venereol Leprol 2009; 75: 187-9.

10. Fagiolo U, Cancian M, Bertollo L, et al. Inhibitory effect of heparin on skin reactivity to autologous serum in chronic idiopathic urticaria. J Allergy Clin Immunol 1999; 103: 1143-7.

11. Czarnecka-Operacz M, Sadowska-Przytocka A, Jenerowicz D, et al. Thyroid function and thyroid autoantibodies in patients with chronic spontaneous urticaria. Adv Dermatol Allergol 2017; 34: 566-72.

12. Sadowska-Przytocka A, Czarnecka-Operacz M, Łącka K, Jenerowicz D. Autoimmune urticaria. Centr Eur J Immunol 2013; 38: 265-70.

13. Asero R. D-dimer: a biomarker for antihistamine-resistant chronic urticaria. J Allergy Clin Immunol 2013; 132: 983-6. 